\title{
Economics of Home Monitoring for Apnea in Late Preterm Infants
}

\author{
Brian L Montenegro MD, Michael Amberson, Lauren Veit MD, Christina Freiberger, \\ Dmitry Dukhovny MD MPH, and Lawrence M Rhein MD MPH
}

\begin{abstract}
BACKGROUND: Apnea of prematurity affects a small proportion but large absolute number of late preterm infants, with out-patient management variably utilized despite relative clinical equipoise and potential for improved cost-effectiveness. METHODS: Over a 5-y period, from 2009 to 2013, infants born at $\geq 34$ weeks gestational age at a level IIIB academic center in Boston, Massachusetts, with discharge-delaying apnea, bradycardia, and desaturation (ABD) events were identified. In-patient costs for discharge-delaying ABD events were compared with hypothetical out-patient management. Outpatient costs took into account 4-10 d of in-patient observation for ABD events before caffeine initiation, 3-5 d of additional in-patient observation before discharge, daily caffeine until 43 weeks corrected gestational age, home pulse oximetry monitoring until 44 weeks corrected gestational age, and consideration of variable readmission rates ranging from 0 to $10 \%$. RESULTS: A total of 425 late preterm and term infants were included in our analysis. Utilization of hypothetical out-patient management resulted in cost savings per eligible patient ranging from $\$ 2,422$ to $\$ 62$, dependent upon variable periods of in-patient observation. Sensitivity analysis demonstrated few instances of decreased relative cost-effectiveness. CONCLUSIONS: Out-patient management of discharge-delaying ABD events in a late preterm and term population was a cost-effective alternative to prolonged in-patient observation. Key words: cost; apnea; bradycardia; desaturation; home monitor; late preterm. [Respir Care 2017;62(1):42-42-48. () 2017 Daedalus Enterprises]
\end{abstract}

\section{Introduction}

Following the acute stabilization of an ill or preterm infant, transition from the neonatal ICU (NICU) to home

\footnotetext{
Dr Montenegro is affiliated with the Division of Newborn Medicine, Boston Children's Hospital, Boston, Massachusetts. Mr Amberson is affiliated with the Boston University School of Medicine, Boston, Massachusetts. Dr Veit is affiliated with the Division of Medicine, Boston Children's Hospital, Boston, Massachusetts. Ms Freiberger is affiliated with Boston College, Chestnut Hill, Massachusetts. Dr Dukhovny is affiliated with the Department of Pediatrics, Oregon Health and Science University, Portland, Oregon. Dr Rhein is affiliated with the Department of Pediatrics, UMass Memorial Children's Medical Center, Worcester, Massachusetts.
}

Dr Montenegro presented a version of this paper at the 41st New England Conference on Perinatal Research, held October 5, 2015, in Chatham, Massachusetts.

The authors have disclosed no conflicts of interest.

Correspondence: Lawrence M Rhein MD MPH, Department of Pediatrics, UMass Memorial Children's Medical Center, Worcester, Massachusetts 01605. E-mail: Lawrence.Rhein@umassmemorial.org.

DOI: $10.4187 /$ respcare.04954 is dependent upon the achievement of a variety of nonacute but discharge-delaying milestones, including the maintenance of temperature stability, feeding maturity, lung maturity, and the resolution of apnea of prematurity. ${ }^{1}$ When present, apnea of prematurity predictably but variably resolves from 34 to 44 weeks gestational age, frequently resulting in a prolonged length of stay (LOS) long after the aforementioned discharge criteria have otherwise been met. ${ }^{2,3}$ Inter-unit variability in the definition and management of apnea of prematurity results in both medical and economic uncertainty in the context of a relatively low acuity condition. ${ }^{4,5}$

The high cost of managing low acuity conditions in an intensive care setting has been recognized in the adult, pediatric, and neonatal intensive care literature. However, resource utilization in newborn medicine is uniquely characterized by a relative lack of alternative settings for nonroutine newborn care. ${ }^{6-9}$ Cost reduction strategies in neonatal intensive care have historically been averse to restrictions in intensity with attempts at reductions in the duration of in-patient care proposed although not widely utilized. ${ }^{10-12}$ The economy of a prolonged in-patient stay, in the setting of apnea of prematurity, has been addressed 
previously. Using computer simulation and varying days of in-patient stay, a relatively decreased cost-effectiveness for infants born at $>30$ weeks gestational age was noted. Specifically, each additional day of monitoring was estimated to cost from $\$ 25,000$ per quality-adjusted life year saved for the first day to $\$ 375,000$ per additional qualityadjusted life year saved for the 10th day. ${ }^{13}$ Such findings highlight the need for tailored management of apnea of prematurity in consideration of, among other factors, gestational age at birth.

Home monitor use in conjunction with caffeine administration serves as one potential avenue for the safe and relatively early discharge of infants with persistent apnea of prematurity. Data on home monitor use in preterm infants with persistent apnea, however, have failed to demonstrate a decrease in LOS. ${ }^{14}$ Such findings must be interpreted with caution, given the retrospective nature of prior studies, the use of home monitors as a secondary option after prolonged in-patient observation, and the exclusive focus on infants born at $<34$ weeks gestational age. ${ }^{4,14-17}$

In fact, a low percentage but large absolute number of infants with apnea of prematurity are born late preterm, defined as birth between $34 \%$ and $36 \% / 7$ weeks gestation, with clinical management specific to this vulnerable population lacking. Yearly, $>300,000$ infants in the United States are born late preterm, with $4-12 \%$ affected by apnea of prematurity. ${ }^{18,19}$

We sought to examine the economic implications of in-patient observation versus hypothetical application of early discharge with home monitoring and out-patient caffeine for a population of late preterm and term infants with discharge-delaying apnea, bradycardia, and desaturation (ABD) events.

\section{Methods}

\section{Patient Population}

Over a 5-y period between January 1, 2009 and December 31,2013 , the medical records of select neonates admitted to Beth Israel Deaconess Medical Center (BIDMC) (Boston, Massachusetts), a large, academic, tertiary center, were reviewed. We identified all infants born at gestational age $\geq 34 \%$ weeks with an ABD event recorded in the electronic medical record $<10 \mathrm{~d}$ before the infants' discharge date. Only infants for whom ABD events remained as the last discharge-delaying diagnosis were included in our analysis. Local practice during the study period required documentation of 5 consecutive ABD-free days before discharge. In addition, clinical practice demanded direct NICU admission for all infants born at $<36$ weeks gestational age. Bradycardic and desaturation events were included in our analysis to account for the

\section{QUICK LOOK}

\section{Current knowledge}

A large proportion of infants born at $<34$ weeks gestational age experience apnea of prematurity. This fact has historically resulted in an academic focus on the moderately to very preterm infant population. Still, apnea of prematurity affects a small proportion but large absolute number of late preterm infants with little data available to guide clinical management. Home monitoring for discharge-delaying apnea of prematurity, as an alternative to prolonged in-patient observation, is variably utilized despite relative clinical equipoise and American Academy of Pediatrics support for selective home monitoring as an appropriate management option.

\section{What this paper contributes to our knowledge}

Home monitoring until 44 weeks corrected gestational age for discharge-delaying apneic, bradycardic, and desaturation events in a late preterm and term population was a cost-effective management option. The degree of cost savings was modulated by variable periods of inpatient observation before the initiation of out-patient management. Longer periods of in-patient observation resulted in decreased relative cost-effectiveness of the out-patient management option.

clinical impact of such events in delaying discharge as well as variable sensitivity of apnea monitoring in the setting of short breathing pauses with only desaturation and/or bradycardia alarms triggered. ${ }^{20}$

Standard definitions for apnea, bradycardia, or desaturation were not utilized in this observational study. Documentation of ABD events was based on the nursing report and physician discretion in accordance with clinical practice at BIDMC. For reference purposes, however, monitor settings during the study period included alarms for apnea $\geq 20 \mathrm{~s}$, heart rate $<80$ beats/min, and pulse oximetry $<90 \%$.

Exclusion criteria included the presence of an alternative etiology for ABD events, such as neurologic or anatomic anomalies (eg, seizures, stroke, Dandy-Walker malformation), severe congenital heart disease (eg, tetralogy of Fallot, transposition of the great arteries), residual lung disease requiring any form of supplementary air flow or oxygen, infection (eg, sepsis, meningitis, urinary tract infection), genetic anomalies, significant dysmorphic features, and drug withdrawal. This study was reviewed and approved with waiver of consent by the institutional review board of BIDMC. 


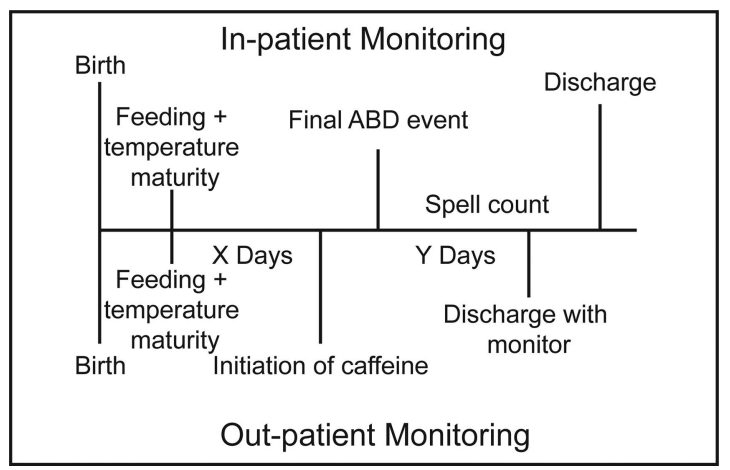

Fig. 1. Standard of care and comparison group. $A B D=$ apnea, bradycardia, desaturation.

\section{Standard of Care and Comparison Group}

Consistent with standard practice, infants at BIDMC with persistent $\mathrm{ABD}$ events were observed until completion of a 5-d spell count. The term "spell count," alternatively labeled "margin of safety for discharge," is defined as several consecutive days before discharge whereby no ABD events are noted..$^{21}$ Alternatively, and although not a part of routine BIDMC clinical practice during the study period, following establishment of $\mathrm{ABD}$ events as the last discharge-delaying diagnoses, caffeine treatment may be initiated or reinitiated in anticipation of out-patient management. The infant is then observed for several days before discharge to ensure resolution of events with caffeine administration and the absence of adverse effects, such as gastroesophageal reflux or tachycardia (Fig. 1). ${ }^{17}$

\section{Comparator}

In-patient costs were calculated from the time when $\mathrm{ABD}$ events were determined to be the sole dischargedelaying diagnosis. Accordingly, following the last day of non-oral feedings and temperature support, in-patient costs were accounted for with all prior in-patient days excluded from the cost analysis. For out-patient management, 2 inpatient parameters were first taken into account, denoted as $\mathrm{X}$ and $\mathrm{Y}$ in Figure 1. First, days spent as an in-patient for discharge-delaying ABD events, and before the initiation of caffeine in anticipation of out-patient management, were varied from 4 to $10 \mathrm{~d}$. Second, a subsequent in-patient period of observation following the initiation of caffeine, but before discharge home, was varied from 3 to $5 \mathrm{~d}$. Both in-patient parameters were derived from local practice. Out-patient costs additionally accounted for daily caffeine until 43 weeks corrected gestational age and daily home monitoring, in the form of out-patient pulse oximetry, until 44 weeks corrected gestational age. Discontinuation of caffeine and out-patient pulse oximetry at 43 and 44 weeks corrected gestational age, respectively, were cho- sen as out-patient management end points in accordance with the largest available home monitoring data set. Namely, the Collaborative Home Infant Monitoring Evaluation (CHIME) study group found no significant differences in apneic events between previously symptomatic preterm and healthy term infants at 44 weeks corrected gestational age. ${ }^{3}$

\section{Sources of Costs}

Four primary costs were taken into consideration: (1) the cost of daily NICU hospitalization; (2) daily out-patient caffeine; (3) daily out-patient pulse oximetry; and (4) the cost of readmission for an apparently life-threatening event. The daily cost of NICU hospitalization was estimated for an infant receiving room air utilizing previously published data regarding the per diem hospital fee, ${ }^{22}$ with the daily Centers for Medicare and Medicaid Services physician payment for convalescent care ${ }^{23}$ additionally taken into account for a total daily cost of $\$ 941 / \mathrm{d}$ of NICU stay. Personal communication with the local BIDMC pharmacy and Boston Children's Hospital case management yielded daily out-patient costs for caffeine and pulse oximetry, respectively. Readmission costs were extrapolated using the literature for apparently life-threatening events, a diagnosis felt to be most consistent with our patient population's risk for readmission. ${ }^{24}$ We conservatively assumed a cost/charge ratio of 0.4 to generate an estimated cost of $\$ 7,427$ per readmission (all costs and sources are noted in Appendix 1; see the supplementary materials at http://www.rcjournal.com). All costs were expressed in 2013 United States dollars, using the consumer price index where necessary to inflate to the same currency.

\section{Sensitivity Analysis}

Sensitivity analyses were conducted at the extremes of out-patient management variables, and thus extremes of cost savings, with each cost parameter adjusted from 50 to $400 \%$ of baseline values. Additionally, given our local experience with persistent and out-patient managed apnea of prematurity in $>150$ infants from 2011 to 2015, we assumed a $0 \%$ rate of readmission for our base case analysis. Published data pertaining to readmission rates for infants discharged with out-patient pulse oximetry and caffeine are currently unavailable. A large review of readmission statistics for all late preterm infants from 2003 to 2012 by Kaiser Permanente of Northern California was remarkable for a $<1 \%$ readmission rate for apparently life-threatening events. ${ }^{25}$ Still, acknowledging that a population of previously symptomatic late preterm and term infants with out-patient pulse oximetry may be at increased risk for readmission secondary to an apparently life-threatening event, we varied readmission rates from 0.5 to $10 \%$. 


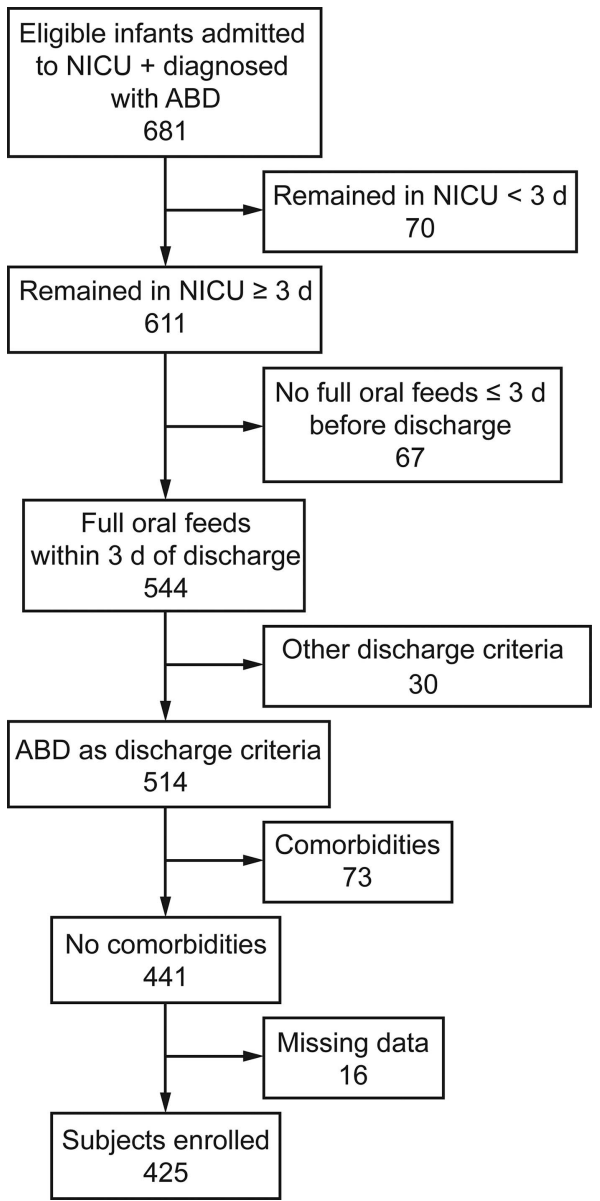

Fig. 2. Flow chart. NICU = neonatal ICU, ABD = apnea, bradycardia, desaturation.

\section{Results}

A total of 681 infants were screened during the 5-y study period, with 425 infants included in our analysis (Fig. 2). The median gestational age at birth of infants included in our study was $354 / 7$ weeks with an interquartile range of $345 / 7$ to $366 / 7$ weeks gestation. The mean $\pm \mathrm{SD}$ LOS for our study cohort was $14.4 \pm 8.3 \mathrm{~d}$ with a mean \pm SD LOS following achievement of full oral feeds and temperature regulation of $9.8 \pm 5.9 \mathrm{~d}$. Proportionally, LOS solely for discharge-delaying events was $\leq 5 \mathrm{~d}$ for $23 \%$ of infants and $>14 \mathrm{~d}$ for $15 \%$ of infants (Fig. 3).

Estimated in-patient costs secondary to discharge-delaying ABD events were $\$ 3,942,790$ for the entire cohort, with individual patient costs ranging from $\$ 2,823$ to $\$ 35,758$. Hypothetical out-patient management yielded 3 potential scenarios: (1) infants whose relatively short stay for $A B D$ events precluded initiation of out-patient management; (2) infants whose stay for ABD events was slightly longer, although it resulted in increased costs with outpatient management; and (3) infants who experienced a

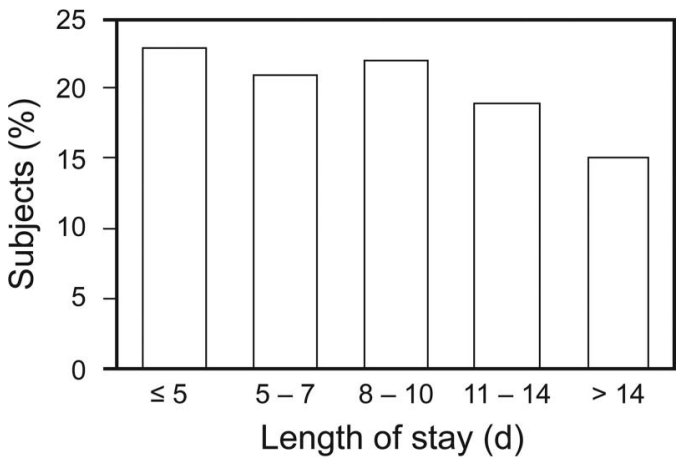

Fig. 3. Length of stay for subjects with apnea, bradycardia, desturation $(\mathrm{ABD})$ events only.

prolonged length stay for $\mathrm{ABD}$ events with notable cost savings upon hypothetical application of out-patient management. The total number of infants that qualified for out-patient management progressively decreased as days to caffeine initiation were varied from 4 to 10 following feeding maturity, temperature regulation, and establishment of ABD events as the sole discharge-delaying diagnosis. Cohort level cost savings were noted across all $\mathrm{X}$ and $\mathrm{Y}$ variable in-patient parameters and ranged from $\$ 874,476$ if caffeine was offered after $4 \mathrm{~d}$ of dischargedelaying ABD events with 3 subsequent days of in-patient observation to $\$ 9,143$ if caffeine was offered after $10 \mathrm{~d}$ of discharge-delaying ABD events with 5 subsequent days of in-patient observation (Fig. 4A). Alternatively, and considering only infants in scenarios 2 and 3 that were eligible for out-patient management, cost savings per eligible patient ranged from $\$ 2,422$ if caffeine was offered after $4 \mathrm{~d}$ of discharge-delaying ABD events with 3 subsequent days of in-patient observation to $\$ 62$ if caffeine was offered after $10 \mathrm{~d}$ of discharge-delaying $\mathrm{ABD}$ events with 5 subsequent days of in-patient observation (Fig. 4B).

\section{Sensitivity Analysis}

Generally, increasing estimates of in-patient costs resulted in economically favorable early discharge, whereas increasing estimates of out-patient costs resulted in economically favorable in-patient management. More specifically, cost savings persisted if caffeine was offered after $4 \mathrm{~d}$ of discharge-delaying ABD events with 3 subsequent days of in-patient observation, with the exception of adjustment of out-patient caffeine to $400 \%$ of the estimated base value. Assuming readmission rates of $0.5,1$, and $10 \%$, cost savings per eligible subject continued under the aforementioned in-patient observation periods. Conversely, greater relative cost of out-patient management was noted in 9 of 15 cases of the adjusted analysis when caffeine was offered after $10 \mathrm{~d}$ of discharge-delaying ABD events with 5 subsequent days of in-patient observation (Table 1). 

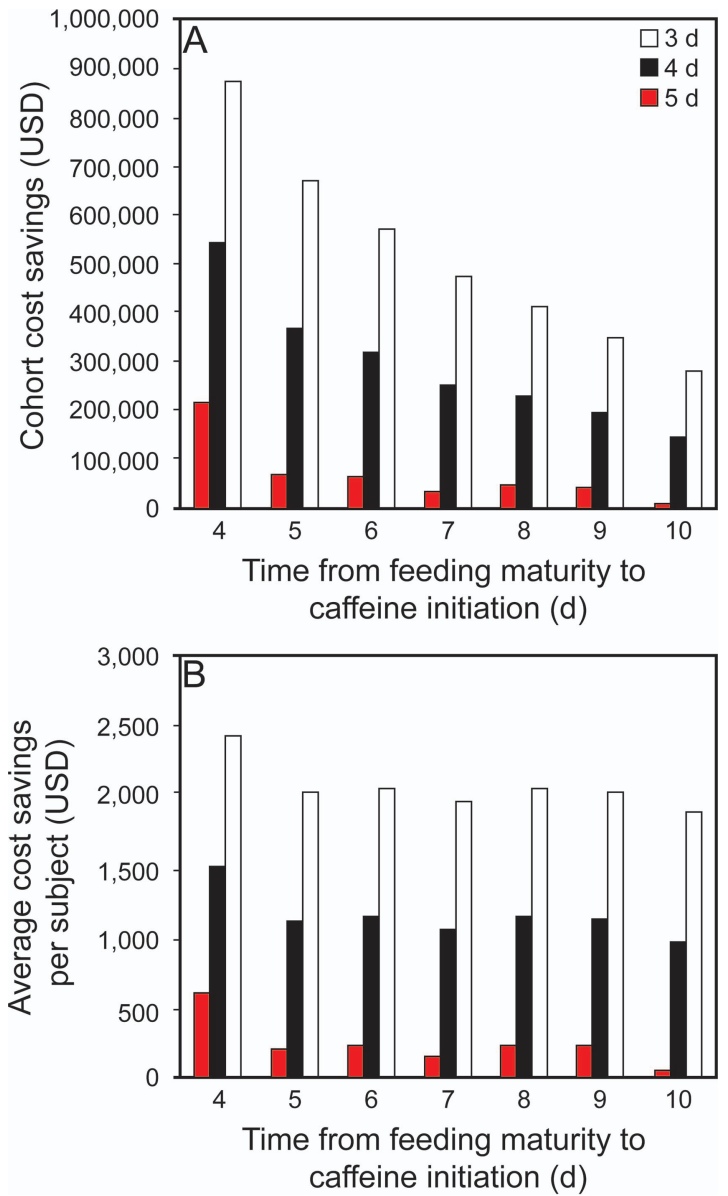

Fig. 4. Cohort level cost savings progressively declined with increasing observational periods (A). Infants were considered for cost analysis only if eligible for out-patient management secondary to persistent apnea, bradycardia, desaturation (ABD) events. Lack of a smooth decline in cost savings with increasing observational periods is accounted for by the variable number of infants considered for cost analysis at each time period (B).

\section{Discussion}

Despite available observational data suggestive of clinical equipoise, the use of home monitors and caffeine, as an alternative to prolonged in-patient observation for persistent $\mathrm{ABD}$ events, remains dependent upon unit-specific practice patterns. ${ }^{4,5,21}$ Our study was able to demonstrate the potential for significant cost savings with out-patient management of discharge-delaying ABD events. Cohort level cost savings were universally noted when out-patient management was offered after 4-10 d of discharge-delaying ABD events with 3-5 subsequent days of in-patient observation before discharge. Sensitivity analysis mostly resulted in persistent cost savings per eligible subject. However, increasing in-patient observational periods resulted in decreased cost benefit, consistent with previous data. ${ }^{13}$

Of importance when considering observational period variations is a notable absence of definitive data in support
Table 1. Sensitivity Analysis Cost Savings per Eligible Subject

\begin{tabular}{lcc}
\hline \hline \multicolumn{1}{c}{ Parameters } & $\begin{array}{c}4 \mathrm{~d} \text { to Caffeine } \\
\text { Initiation/3 d } \\
\text { In-Patient Observation } \\
(n=360)\end{array}$ & $\begin{array}{c}10 \mathrm{~d} \text { to Caffeine } \\
\text { Initiation/5 d } \\
\text { In-Patient Observation } \\
(n=148)\end{array}$ \\
\hline Base case* & 2,422 & 62 \\
Hospital cost & 566 & -536 \\
$50 \%$ & 6,134 & 1,257 \\
$200 \%$ & 13,558 & 3,648 \\
$400 \%$ & & \\
Home monitor cost & 2,622 & 2,071 \\
$50 \%$ & 2,022 & -290 \\
$200 \%$ & 1,222 & -994 \\
$400 \%$ & & \\
Out-patient caffeine cost & 2,867 & 453 \\
$50 \%$ & 1,533 & -720 \\
$200 \%$ & -246 & $-2,283$ \\
$400 \%$ & & \\
Readmission rate & 2,385 & -12 \\
$0.5 \%$ & 2,348 & -681 \\
$1 \%$ & 1,680 & \\
$10 \%$ & & -12 \\
Readmission cost (0.5\% & & \\
readmission rate $)$ & 2,404 & \\
$50 \%$ & 2,348 & \\
$200 \%$ & 2,274 & \\
$400 \%$ & & \\
& & \\
\hline All values are in United States dollars. & & \\
Base case analysis with 0\% readmission rate based on local experience. & \\
\hline
\end{tabular}

of a defined safe period of in-patient observation. ${ }^{26,27}$ In the only available survey of $>300$ neonatologists, published in 1997, responding to the question of a margin of safety, responses varied from 1 to $10 \mathrm{~d}$. Approximately 75\% supported a 5-7-d event-free observation period before discharge. ${ }^{21}$ Most recently, in 2016, a clinical report on apnea of prematurity by the American Academy of Pediatrics advocated for an individual approach to discharge management considering gestational age at birth as well as the severity and nature of events. ${ }^{27}$

Of additional importance for consideration is the method of reimbursement assumed. In the midst of our study period, the passage of the Patient Protection and Affordable Care Act of 2010 required a shift in the reimbursement paradigm from a predominately fee-for-service to a bundled payment model. ${ }^{28}$ The impact on neonatal care remains vague, with concerns abounding and including the potential for destabilization of regional perinatal referral systems, underuse of essential services in the setting of capitation, and suboptimal care of costly outlier cases. ${ }^{29}$ Under this new model of health care, savings may be realized via utilization efficiency rather than fee-for-service cost savings.

Regardless of whether the cost of out-patient monitoring is superior or even equivalent to in-patient observation, consideration of relative quality of life is important. Where 
appropriate, parental involvement in the care of NICU patients has previously been demonstrated to effectively decrease stress. ${ }^{30}$ The potential for increased stress with home monitoring is undeniable, although pertinent studies have yielded conflicting results. ${ }^{31-35}$

Moreover, our study assumed the safety of home monitor use with out-patient caffeine for persistent apnea of prematurity. In the absence of randomized prospective data specifically pertaining to the safety of out-patient pulse oximetry use and caffeine for persistent apnea of prematurity, providers may be disinclined to utilize an out-patient management option. Still, targeted home monitor use is supported by the most recent American Academy of Pediatrics clinical report on apnea of prematurity. ${ }^{27} \mathrm{We}$ also assumed that all infants initiated on caffeine would cease to experience further ABD events. A small proportion of infants, however, may not respond to caffeine treatment and would require further in-patient management. Even following discharge with out-patient pulse oximetry and caffeine, parental expenditures and days lost from work may have the unintended consequence of costshifting from hospitals and/or insurers to caregivers. ${ }^{36}$ Furthermore, our study did not utilize standard definitions for ABD events, and documentation was based on the nursing report and clinician discretion, consistent with clinical practice. The lack of routine cardiorespiratory monitor review invites the possibility of misclassification of $A B D$ events. ${ }^{37,38}$ Last, extrapolation of our theoretical model is limited because our data were derived from infants at a single level IIIB academic center.

Despite these study limitations, our retrospective cost analysis demonstrates the potential for significant cost savings when out-patient management for persistent ABD events is offered to a cohort of affected late preterm and term infants. The absence of a unified approach for the clinical management of persistent $\mathrm{ABD}$ events presents the opportunity for both quality improvement and significant cost savings. Confirmation of our findings via a multisite and prospective study design is required to further examine the potential for out-patient pulse oximetry use as a safe, family-centered, and cost-effective management option for late preterm and term infants with persistent $\mathrm{ABD}$ events.

\section{REFERENCES}

1. American Academy of Pediatrics Committee on Fetus and Newborn. Hospital discharge of the high-risk neonate. Pediatrics 2008;122(5): 1119-1126.

2. Eichenwald EC, Aina A, Stark AR. Apnea frequently persists beyond term gestation in infants delivered at 24 to 28 weeks. Pediatrics 1997;100(3 Pt 1):354-359.

3. Ramanathan R, Corwin MJ, Hunt CE, Lister G, Tinsley LR, Baird T, et al. Cardiorespiratory events recorded on home monitors: Comparison of healthy infants with those at increased risk for SIDS. JAMA 2001;285(17):2199-2207.
4. Eichenwald EC, Blackwell M, Lloyd JS, Tran T, Wilker RE, Richardson DK. Inter-neonatal intensive care unit variation in discharge timing: influence of apnea and feeding management. Pediatrics 2001; 108(4):928-933.

5. Eichenwald EC, Zupancic JA, Mao WY, Richardson DK, McCormick MC, Escobar GJ. Variation in diagnosis of apnea in moderately preterm infants predicts length of stay. Pediatrics 2011;127(1): e53-e58.

6. Singer DE, Carr PL, Mulley AG, Thibault GE. Rationing intensive care: physician responses to a resource shortage. N Engl J Med 1983;309(19):1155-1160.

7. Strauss MJ, LoGerfo JP, Yeltatzie JA, Temkin N, Hudson LD. Rationing of intensive care unit services: an everyday occurrence. JAMA 1986;255(9):1143-1146.

8. Pollack MM, Getson PR, Ruttimann UE, Steinhart CM, Kanter RK, Katz RW, et al. Efficiency of intensive care: a comparative analysis of eight pediatric intensive care units. JAMA 1987;258(11):14811486.

9. Zupancic JA, Richardson DK. Characterization of the triage process in neonatal intensive care. Pediatrics 1998;102(6):1432-1436.

10. Richardson DK, Zupancic JA, Escobar GJ, Ogino M, Pursley DM, Mugford M. A critical review of cost reduction in neonatal intensive care. II. Strategies for reduction. J Perinatol 2001;21(2):121-127.

11. Merritt TA, Pillers D, Prows SL. Early NICU discharge of very low birth weight infants: a critical review and analysis. Semin Neonatol 2003;8(2):95-115.

12. Collins CT, Makrides M, McPhee AJ. Early discharge with home support of gavage feeding for stable preterm infants who have not established full oral feeds. Cochrane Database Syst Rev 2003;(4): CD003743.

13. Zupancic JA, Richardson DK, O'Brien BJ, Eichenwald EC, Weinstein MC. Cost-effectiveness analysis of predischarge monitoring for apnea of prematurity. Pediatrics 2003;111(1):146-152.

14. Perfect Sychowski S, Dodd E, Thomas P, Peabody J, Clark R. Home apnea monitor use in preterm infants discharged from newborn intensive care units. J Pediatr 2001;139(2):245-248.

15. Côté A, Hum C, Brouillette RT, Themens M. Frequency and timing of recurrent events in infants using home cardiorespiratory monitors. J Pediatr 1998;132(5):783-789.

16. Lorch SA, Srinivasan L, Escobar GJ. Epidemiology of apnea and bradycardia resolution in premature infants. Pediatrics 2011;128(2): e366-e373.

17. Butler TJ, Firestone KS, Grow JL, Kantak AD. Standardizing documentation and the clinical approach to apnea of prematurity reduces length of stay, improves staff satisfaction, and decreases hospital cost. Jt Comm J Qual Patient Saf 2014;40(6):263-269.

18. Martin JA, Hamilton BE, Osterman MJ, Curtin SC, Matthews TJ. Births: final data for 2013. Natl Vital Stat Rep 2015;64(1):1-68.

19. Engle WA, Tomashek KM, Wallman C. "Late-preterm" infants: a population at risk. Pediatrics 2007;120(6):1390-1401.

20. Di Fiore JM, Arko MK, Miller MJ, Krauss A, Betkerur A, Zadell A, et al. Cardiorespiratory events in preterm infants referred for apnea monitoring studies. Pediatrics 2001;108(6):1304-1308.

21. Darnall RA, Kattwinkel J, Nattie C, Robinson M. Margin of safety for discharge after apnea in preterm infants. Pediatrics 1997;100(5): 795-801.

22. Zupancic JA, Hibbs AM, Palermo L, Truog WE, Cnaan A, Black DM, et al. Economic evaluation of inhaled nitric oxide in preterm infants undergoing mechanical ventilation. Pediatrics 2009;124(5): 1325-1332.

23. Center for Medicare and Medicare Services. Physician fee schedule. www.cms.gov/Medicare/Medicare-Fee-for-Service-Payment/ PhysicianFeeSched/index.html. Accessed July 1, 2015. 


\section{Economics of Home Apnea Monitoring}

24. Tieder JS, Cowan CA, Garrison MM, Christakis DA. Variation in inpatient resource utilization and management of apparent life-threatening events. J Pediatr 2008;152(5):629-635, 635. e1-635.e2.

25. Kuzniewicz MW, Parker SJ, Schnake-Mahl A, Escobar GJ. Hospital readmissions and emergency department visits in moderately preterm, late preterm, and early preterm infants. Clin Perinatol 2013; 40(4):753-775.

26. Finer NN, Higgins R, Kattwinkel J, Martin RJ. Summary proceedings from the apnea-of-prematurity group. Pediatrics 2006;117(3 Pt 2):S47-S51.

27. Eichenwald EC and Committee on Fetus and Newborn. Apnea of prematurity. Pediatrics 2016;137(1):1-7.

28. American Hospital Association. Issue brief: moving towards bundled payment. 2013. www.aha.org/content/13/13jan-bundlingissbrief.pdf. Accessed November 2, 2016.

29. Profit J, Wise PH, Lee HC. Consequences of the affordable care act for sick newborns. Pediatrics 2014;134(5):e1284-e1286.

30. Heermann JA, Wilson ME, Wilhelm PA. Mothers in the NICU: outsider to partner. Pediatr Nurs 2005;31(3):176-181, 200.
31. Black L, Hersher L, Steinschneider A. Impact of the apnea monitor on family life. Pediatrics 1978;62(5):681-685.

32. Wasserman AL. A prospective study of the impact of home monitoring on the family. Pediatrics 1984;74(3):323-329.

33. McElroy E, Steinschneider A, Weinstein S. Emotional and health impact of home monitoring on mothers: a controlled prospective study. Pediatrics 1986;78(5):780-786.

34. Vohr BR, Chen A, Garcia Coll C, Oh W. Mothers of preterm and full-term infants on home apnea monitors. Am J Dis Child 1988;142(2): 229-231.

35. Leonard BJ, Scott SA, Sootsman J. A home-monitoring program for parents of premature infants: a comparative study of the psychological effects. J Dev Behav Pediatr 1989;10(2):92-97.

36. Donath S. Hospital in the home: real cost reductions or merely cost-shifting? Aust N Z J Public Health 2001;25(2):187-188.

37. Muttitt SC, Finer NN, Tierney AJ, Rossmann J. Neonatal apnea: diagnosis by nurse versus computer. Pediatrics 1988;82(5):713-720.

38. Vagedes J, Poets CF, Dietz K. Averaging time, desaturation level, duration and extent. Arch Dis Child Fetal Neonatal Ed 2013;98(3):F265-F266.

This article is approved for Continuing Respiratory Care Education credit. For information and to obtain your CRCE

(free to AARC members) visit

www.rcjournal.com 